\title{
Changes in the plasma levels of cytokines/chemokines for predicting the response to chemoradiation therapy in rectal cancer patients
}

\author{
NORIKO TADA ${ }^{1}$, NELSON H. TSUNO ${ }^{1,2}$, KAZUSHIGE KAWAI $^{1}$, KOJI MURONO $^{1}$, TAKAKO NIREI ${ }^{1}$, \\ SOICHIRO ISHIHARA ${ }^{1}$, EIJI SUNAMI ${ }^{1}$, JOJI KITAYAMA ${ }^{1}$ and TOSHIAKI WATANABE ${ }^{1}$
}

Departments of ${ }^{1}$ Surgical Oncology, ${ }^{2}$ Transfusion Medicine, Faculty of Medicine, University of Tokyo, Tokyo 113-0033, Japan

Received September 10, 2013; Accepted October 14, 2013

DOI: $10.3892 /$ or.2013.2857

\begin{abstract}
In the present study, we aimed to characterize the predictive value of cytokines/chemokines in rectal cancer (RC) patients receiving chemoradiation therapy (CRT). Blood samples were obtained pre- and post-CRT from 35 patients with advanced RC, who received neoadjuvant CRT followed by surgery, and the correlation between plasma levels of cytokines/chemokines and the response to CRT was analyzed. The pre-CRT levels of soluble CD40-ligand (sCD40L) and the post-CRT levels of chemokine ligand-5 (CCL-5) were significantly associated with the depth of tumor invasion and with venous invasion. In addition, a significant decrease in SCD40L and CCL-5, as well as in platelet counts, was associated with a favorable response to CRT. A significant correlation between pre-CRT platelet counts and SCD40L was observed in patients with a favorable response. By contrast, higher post-CRT interleukin (IL)-6 was associated with a poor response. Platelets, immune system and cancer cells, cross-linked through various cytokines/chemokines, appear to play an important role in the response to CRT, and by understanding their roles, new approaches for the improvement of the therapy might be proposed.
\end{abstract}

\section{Introduction}

Surgical resection is one of the most reliable treatment modalities for rectal cancer ( $\mathrm{RC}$ ), which is one of the commonest malignancies in the world. At present, concurrent chemoradiation therapy (CRT) is the standard treatment modality, which, when administered preoperatively, is useful for tumor downstaging, for increasing the curative resection rate, and for decreasing the local recurrence rate (1-3). There is, however,

Correspondence to: Dr Noriko Tada, Department of Surgical Oncology, University of Tokyo, 7-3-1 Hongo, Tokyo 113-0033, Japan E-mail: tadanoriko520317@yahoo.co.jp

Key words: chemoradiation therapy, rectal cancer, cytokines inter-individual variability in the response to CRT, thus, in order to avoid its indication for those who will not benefit from it, it is desirable to identify the individuals who are less sensitive to CRT. However, at present, no effective markers of the response to CRT have been reported.

The response to CRT depends not only on the direct injury of cancer cells by chemotherapeutic agents and/or the radiation, but also on the tumor antigen-specific T-cell responses elicited by the injured cancer cells. It is well-known that the biological behavior of cancer cells, or the tumor microenvironment, which includes inflammatory cells and cytokines, might determine, or at least influence, the response to therapy (4-6).

The immune system is divided into innate and adaptive, which extensively interact (crosstalk) with each other. The innate response includes several cellular effectors, such as natural killer (NK) cells, macrophages, dendritic cells (DCs), and plays an important role as the first line of defense. Adaptive immunity is a more specific response, which is mediated by antibodies and helper [CD4(+)] and cytotoxic [CD8(+)] T-cells. Both innate and adaptive immune responses play an important role in antitumor immunity. Recently, the existence of cells with suppressive effects on the immune system, namely regulatory T-cells (Tregs) and myeloid-derived suppressor cells (MDSCs), which play important roles in the escape of cancer cells from the host immune response, thus contributing to the development and progression of cancer, has been reported $(7,8)$. Therefore, the balance between the antitumor effector cells and the immunosuppressive cells is important in determining the response to therapy.

Generally, cancer cells and immune cells interact with each other through direct cell-cell contact or by production of soluble factors, such as cytokines. Cancer cells are known as an important source of inflammatory cytokines/growth factors, and the production of different cytokines in the tumor microenvironment determines cancer behavior. Alternatively, cytokines can be produced by effector cells that promote antitumor immunity, inhibiting tumor development and progression. By contrast, cytokines that promote tumor growth, attenuate apoptosis and facilitate invasion and metastasis can be produced by host immune cells (6,9-11). An endocrine loop between cancer cells, effector and suppressor cells, crosslinked by tumor-derived soluble factors, such as transforming 
growth factor- $\beta$ (TGF- $\beta$ ) and interleukin (IL)-10, can generate a potent immunoinhibitory effect on the antitumor immune response, promoting cancer cell survival and proliferation. Thus, this complex interaction between cancer cells and the host immune system, involving production of cytokines, may play an important role in the response to CRT.

In a recent report (Tada et al, unpublished data), we investigated the predictive value of host immune cells, especially lymphocyte subsets, in the response of RC to CRT, and found that the pre-CRT counts of T-lymphocytes and helper T-lymphocytes significantly correlated with the response to CRT.

In the present study, we aimed to investigate the concentrations of various cytokines in the plasma of RC patients receiving CRT, and their correlation with the response to therapy, in an attempt to find potential predictive markers of the response to CRT, which would help the selection of those patients who would or would not benefit from receiving therapy, and to understand the complex interaction between cancer cells and the tumor microenvironment, mediated by various cytokines.

\section{Materials and methods}

Patients. The study protocol was approved by the local ethics committee, and written informed consent was obtained from all patients. The studied population included 35 consecutive patients with primary RC of clinical stage T3-4 and M0, receiving treatment at the Department of Surgical Oncology, the University of Tokyo Hospital, in the period between January 2010 and August 2012.

The enrolled patients received a total dose of $50 \mathrm{~Gy}$ of radiation and concomitant 5-FU-based chemotherapy, followed by standardized curative resection, applied in an interval of 6-8 weeks. Peripheral venous blood samples were obtained before neoadjuvant CRT and 4-6 weeks following completion of CRT, prior to surgery.

Flow cytometry. Peripheral blood cells (PBS) were analyzed on the FACSCalibur flow cytometer using the Multiset software package, and the collected data were analyzed using the CellQuest software (all from BD Biosciences, San Jose, CA, USA).

A combination of fluorescein isothiocyanate (FITC) and phycoerythrin (PE)-conjugated monocolonal antibody (BD Biosciences) was used to identify the lymphocyte subsets and the MDSCs, as follows: CD3(+)/CD19(-) for the T-lymphocytes, CD3(-)/CD19(+) for the B-lymphocytes, CD3(-)/CD56(+) for the NK cells, and CD33(+)/HLA(-)DR(-) for MDSCs (Table I).

Biochemical determination. The serum samples were clarified by spinning at $3,000 \mathrm{rpm}$ for $10 \mathrm{~min}$ at $20-25^{\circ} \mathrm{C}$, and immediately stored at $-40^{\circ} \mathrm{C}$ prior to biochemical analyses. The serum concentration of IL-2, IL-4, IL-6, IL-10, interferon (IFN)- $\gamma$, tumor necrosis factor (TNF)- $\alpha$, monocyte-chemoattractant protein (MCP)-1, chemokine ligand-5 [(CCL-5, previously known as regulated on activation normal T-cell expressed and secreted (RANTES)], tumor necrosis factor-related apoptosis inducing ligand (TRAIL) and soluble CD40-ligand (sCD40L)
Table I. Surface marker expression according to the cell phenotype.

\begin{tabular}{ll} 
Surface marker FITC/PE & Leukocyte subset \\
\hline CD3(+)/CD19(-) & T-lymphocytes \\
CD3(-)/CD19(+) & B-lymphocytes \\
CD3(-)/CD56(+) & NK cells \\
CD33(+)/HLA(-)DR(-) & MDSCs
\end{tabular}

FITC, fluorescein isothiocyanate; PE, phycoerythrin; NK cells, natural killer cells; MDSCs, myeloid-derived suppressor cells.

was measured using a Procarta polystyrene bead-based multiplex immunoassay (Affymetrix, Inc., Santa Clara, CA, USA) on the Luminex instrument and the data were analyzed using the Luminex IS2.2/2.3 software.

Histological study. All the resected specimens were histologically examined, and the findings were recorded in accordance with the TMN classification. The histological regression of the primary rectal lesions in response to CRT was evaluated by pathologists, and classified as high or low, based on the amount of residual cancer, according to the Japanese Classification of Colorectal Carcinoma: cases in which more than two-thirds of the cancer had degraded, necrotized, or disappeared were classified as high histological regression (High-R), and those with less than two-third reduction were classified as low histological regression (Low-R).

Statistical Analysis. The analyses of the associations between the serum concentration of cytokines and the clinicopathological variables were carried out using the Mann-Witney $U$ test. The difference in the serum pre- and post-CRT concentration of cytokines was calculated using the Wilcoxon matched paired t-test. All analyses were performed with JMP 9.0 software, and a P-value $<0.05$ was considered to indicate a statistically significant difference.

\section{Results}

The clinicopathological features of the patients are provided in Table II. Among them, 23 (65.7\%) were male and 12 (34.3\%) female. The median age was 62 (range 43-85) years. According to the pathological response to CRT, the patients were divided into two groups, i.e., low response (Low-R) (18 cases, 51.4\%) and high response (High-R) (17 cases, 48.6\%), and the four cases of pathological complete response (CR) were included in the High-R.

First, the correlation between the pre-/post-CRT cytokine levels and clinicopathological factors of the patients was evaluated. A significant correlation between pre-CRT levels of $\mathrm{SCD} 40 \mathrm{~L}$ and the depth of invasion and the venous invasion was observed (Table III), but the correlation was not found in the post-CRT. Cases of T0-2, i.e., those with remarkable dowsizing of $\mathrm{T}$ factor, showed significantly higher concentrations of pre-CRT sCD40L compared to those without (T3-4), and cases without venous invasion had higher levels of sCD40L than those without. Similarly, post-CRT levels of 
Table II. Patient characteristics $(n=35)$.

\begin{tabular}{|c|c|}
\hline Characteristics & No. of patients $(\%)$ \\
\hline \multicolumn{2}{|l|}{ Gender } \\
\hline Male & $23(65.7)$ \\
\hline Female & $12(34.3)$ \\
\hline \multicolumn{2}{|l|}{ Age (years) } \\
\hline Median & 62 \\
\hline Range & $43-85$ \\
\hline \multicolumn{2}{|l|}{ Histological regression } \\
\hline Low-R & $18(51.4)$ \\
\hline High-R & $17(48.6)$ \\
\hline \multicolumn{2}{|l|}{ Depth of tumor } \\
\hline урT0-pT2 & $17(48.6)$ \\
\hline ypT3-pT4 & $18(51.4)$ \\
\hline \multicolumn{2}{|l|}{ Lymph node metastasis } \\
\hline Absent & $24(68.6)$ \\
\hline Present & $11(31.4)$ \\
\hline \multicolumn{2}{|l|}{ Lymphatic invasion } \\
\hline Absent & $31(88.6)$ \\
\hline Present & $4(11.5)$ \\
\hline \multicolumn{2}{|l|}{ Venous invasion } \\
\hline Absent & $14(40.0)$ \\
\hline Present & $21(60.0)$ \\
\hline \multicolumn{2}{|l|}{ Histological classification } \\
\hline Well-differentiated adenocarcinoma & $13(37.1)$ \\
\hline Moderately differentiated adenocarcinoma & $15(42.9)$ \\
\hline Mucinous carcinoma & $1(2.9)$ \\
\hline No cancer remaining & $6(17.1)$ \\
\hline
\end{tabular}

Values represent number (percentage) unless otherwise specified. Low-R, low response; High-R, high response.

CCL-5 significantly correlated with the depth of tumor invasion and with venous invasion, with higher levels found in the ypT3-4 cases compared to ypT0-2 ones, and in those with venous invasion compared to those without (Table III).

Next, the plasma levels of various cytokines were compared between pre- and post-CRT samples, and a significant decrease in the level of sCD40L was observed in post-CRT compared to pre-CRT (Fig. 1). The levels of IL-6 showed a tendency to increase in post-CRT samples, but this result was not statistically significant. There were no significant differences between the pre- and post-CRT concentrations of the other cytokines tested, namely IL-2, IL-4, IL-10, IFN- $\gamma$, TNF- $\alpha$, MCP-1, CCL-5 and TRAIL. Next, the pre- and postCRT levels of the plasma cytokines were analyzed according to the pathological response to CRT, i.e., High-R and Low-R. A significant difference between High-R and Low-R was observed in the post-CRT levels of IL-6 and TNF- $\alpha$, both being higher in Low-R compared to High-R. None of the pre-CRT cytokine levels correlated with the pathological response to CRT (Table IV). Next, we compared the pre- and post-CRT concentration of the plasma cytokines in High-R and Low-R. The mean post-CRT levels of sCD40L and CCL-5 were significantly lower compared to the pre-CRT ones in the High-R, but not in the Low-R (Fig. 2). In addition, postCRT platelet counts were significantly decreased compared to the pre-CRT values in both High-R and Low-R, while the difference was more evident in the High-R. By contrast, the post-CRT level of IL-6 was significantly higher compared to the pre-CRT one in the Low-R, but not in the High-R. No significant differences were observed between the pre- and post-CRT concentrations in samples of different pathological response to CRT for the rest of the cytokines.

Since it is well-known that most sCD40L and CCL-5 are produced by platelets and T-lymphocytes, next, we analyzed the correlation between platelet counts and the plasma levels of sCD40L and CCL-5, pre- and post-CRT, according to the response to CRT. Notably, we found that the pre-CRT sCD40L significantly correlated with platelet counts only in pre-CRT samples of High-R, but not in Low-R (Fig. 3A). No such correlation was found between the post-CRT levels of CD40L and the platelet counts in either group (Fig. 3B). In addition, no correlation was found between the plasma levels of CCL-5 and platelet counts, either pre- or post-CRT, in either High-R or Low-R. As expected, a tendency for a negative correlation between pre-CRT sCD40L and CCL-5 levels was observed only in the High- $\mathrm{R}(\mathrm{R}=-0.36, \mathrm{P}=0.15)$, but not in the Low- $\mathrm{R}$ $(\mathrm{R}=0.02)$. Also, no correlation was observed between postCRT levels of sCD40L and CCL5 in either High-R or Low-R (data not shown).

Next, the involvement of the immune cells in the response to therapy was evaluated in High-R and Low-R. The pre-CRT T-lymphocyte counts in High-R were significantly higher than those in Low-R (Table V), whereas in the post CRT samples, no difference between Low-R and High-R was observed. However, no correlation was found between CD3(+) T-cell counts and sCD40L in either pre- or post-CRT, in either High-R or Low-R. Moreover, there was no correlation between the pre-CRT CCL-5 level and CD3(+) T-cell counts, in either High-R or Low-R (data not shown). The post-CRT CCL-5 level, however, showed a negative, but not significant, correlation with CD3(+) T-cell counts in the High-R $(\mathrm{R}=-0.37$, $\mathrm{P}=0.14$ ), whereas a positive, but not significant, correlation was observed in the Low-R $(\mathrm{R}=0.33, \mathrm{P}=0.18)$ (data not shown).

To evaluate the hypothesis that SCD40L plays an immunosuppressive role, we analyzed the percentage of MDSCs in peripheral blood. No significant change in the percentage of MDSCs was observed post-CRT $(\mathrm{P}=0.26)$, compared to pre-CRT, and also no differences between Low-R and High-R were observed (pre-CRT, $\mathrm{P}=0.31$; post-CRT, $\mathrm{P}=0.91$ ) (Table V). In addition, no correlation between the percentage of MDSCs and the concentration of SCD40L was found.

\section{Discussion}

It was previously believed that the antitumor effects of CRT were almost completely dependent on the direct injury of tumor cells by anticancer agents and/or irradiation, but recently, it was confirmed that the immune system further contributes to the antitumor effects of CRT (12).

Exposure to irradiation leads to an inflammatory response that is highly heterogeneous, but only few reports have analyzed the relevant mechanism, especially related to changes in cyto- 
Table III. Correlations between clinicopathological factors and the cytokine levels.

A, Depth of invasion

\begin{tabular}{|c|c|c|c|c|c|c|}
\hline \multirow[b]{2}{*}{ Cytokines $^{\mathrm{a}}$} & \multicolumn{3}{|c|}{ Pre-CRT } & \multicolumn{3}{|c|}{ Post-CRT } \\
\hline & урТ0-2 & урT3-4 & P-value & урТ0-2 & урT3-4 & P-value \\
\hline IFN- $\gamma$ & $0.69 \pm 0.94$ & $0.17 \pm 0.25$ & $0.038^{\mathrm{b}}$ & $0.34 \pm 0.55$ & $0.21 \pm 0.23$ & 0.369 \\
\hline IL-10 & $1.45 \pm 1.87$ & $0.74 \pm 0.86$ & 0.171 & $1.08 \pm 1.47$ & $1.07 \pm 1.28$ & 0.988 \\
\hline IL-2 & $3.44 \pm 5.43$ & $1.53 \pm 2.53$ & 0.199 & $2.31 \pm 4.26$ & $1.59 \pm 2.52$ & 0.554 \\
\hline IL-4 & $10.40 \pm 13.54$ & $5.21 \pm 6.66$ & 0.167 & $6.68 \pm 7.97$ & $7.02 \pm 9.89$ & 0.911 \\
\hline IL-6 & $11.76 \pm 12.68$ & $8.32 \pm 5.59$ & 0.316 & $44.51 \pm 91.88$ & $23.60 \pm 28.05$ & 0.426 \\
\hline MCP-1 & $33.69 \pm 23.61$ & $38.12 \pm 21.15$ & 0.563 & $34.94 \pm 36.52$ & $35.77 \pm 24.46$ & 0.938 \\
\hline CCL-5 & $1.29 \pm 1.05$ & $1.86 \pm 1.10$ & 0.114 & $1.05 \pm 0.71$ & $1.63 \pm 0.98$ & $0.05^{\mathrm{b}}$ \\
\hline sCD40L & $10.85 \pm 3.79$ & $7.67 \pm 3.24$ & $0.012^{\mathrm{b}}$ & $6.85 \pm 3.22$ & $7.61 \pm 5.03$ & 0.594 \\
\hline $\mathrm{TNF}-\alpha$ & $4.06 \pm 4.98$ & $2.44 \pm 3.27$ & 0.267 & $3.54 \pm 4.01$ & $7.33 \pm 13.68$ & 0.274 \\
\hline TRAIL & $46.56 \pm 26.39$ & $34.18 \pm 11.32$ & 0.109 & $53.04 \pm 73.36$ & $35.01 \pm 13.97$ & 0.349 \\
\hline
\end{tabular}

B, Lymph node metastasis

\begin{tabular}{|c|c|c|c|c|c|c|}
\hline \multirow[b]{2}{*}{ Cytokines $^{\mathrm{a}}$} & \multicolumn{3}{|c|}{ Pre-CRT } & \multicolumn{3}{|c|}{ Post-CRT } \\
\hline & Absent & Present & $\mathrm{P}$-value & Absent & Present & P-value \\
\hline IFN- $\gamma$ & $0.43 \pm 0.73$ & $0.39 \pm 0.72$ & 0.89 & $0.30 \pm 0.45$ & $0.22 \pm 0.33$ & 0.59 \\
\hline IL-10 & $1.01 \pm 1.44$ & $1.25 \pm 1.58$ & 0.68 & $1.01 \pm 1.29$ & $1.22 \pm 1.55$ & 0.69 \\
\hline IL-2 & $2.04 \pm 3.92$ & $3.37 \pm 4.97$ & 0.45 & $1.83 \pm 3.66$ & $2.19 \pm 3.07$ & 0.76 \\
\hline IL-4 & $7.49 \pm 10.77$ & $8.26 \pm 11.20$ & 0.85 & $7.24 \pm 10.04$ & $6.01 \pm 5.91$ & 0.66 \\
\hline IL-6 & $8.83 \pm 9.47$ & $12.54 \pm 10.21$ & 0.32 & $43.38 \pm 78.47$ & $13.30 \pm 14.05$ & 0.11 \\
\hline MCP-1 & $34.98 \pm 25.35$ & $38.13 \pm 13.58$ & 0.64 & $36.29 \pm 35.93$ & $33.35 \pm 13.25$ & 0.73 \\
\hline CCL-5 & $1.54 \pm 1.01$ & $1.62 \pm 1.33$ & 0.87 & $1.22 \pm 0.83$ & $1.63 \pm 1.01$ & 0.27 \\
\hline sCD40L & $9.35 \pm 3.88$ & $8.92 \pm 3.84$ & 0.76 & $7.80 \pm 4.49$ & $6.02 \pm 3.39$ & 0.21 \\
\hline TNF- $\alpha$ & $3.09 \pm 4.20$ & $3.53 \pm 4.41$ & 0.79 & $6.72 \pm 12.02$ & $2.81 \pm 3.57$ & 0.16 \\
\hline TRAIL & $42.30 \pm 23.09$ & $34.89 \pm 12.68$ & 0.25 & $37.75 \pm 17.46$ & $56.01 \pm 88.18$ & 0.51 \\
\hline
\end{tabular}

C, Lymphatic invasion

\begin{tabular}{|c|c|c|c|c|c|c|}
\hline \multirow[b]{2}{*}{ Cytokines $^{\mathrm{a}}$} & \multicolumn{3}{|c|}{ Pre-CRT } & \multicolumn{3}{|c|}{ Post-CRT } \\
\hline & Absent & Present & P-value & Absent & Present & P-value \\
\hline IFN- $\gamma$ & $0.39 \pm 0.67$ & $0.66 \pm 1.12$ & 0.67 & $0.26 \pm 0.41$ & $0.38 \pm 0.55$ & 0.72 \\
\hline IL-10 & $1.01 \pm 1.39$ & $1.68 \pm 2.11$ & 0.58 & $0.92 \pm 1.16$ & $2.30 \pm 2.26$ & 0.31 \\
\hline IL-2 & $2.12 \pm 3.85$ & $5.10 \pm 6.77$ & 0.45 & $1.68 \pm 3.27$ & $3.94 \pm 4.62$ & 0.41 \\
\hline IL-4 & $7.24 \pm 9.87$ & $11.53 \pm 17.71$ & 0.67 & $6.74 \pm 9.05$ & $7.77 \pm 8.54$ & 0.83 \\
\hline IL-6 & $8.99 \pm 8.90$ & $17.72 \pm 13.68$ & 0.29 & $6.47 \pm 69.99$ & $13.11 \pm 12.13$ & 0.13 \\
\hline MCP-1 & $34.79 \pm 22.81$ & $45.06 \pm 13.35$ & 0.29 & $35.40 \pm 32.14$ & $35.09 \pm 13.20$ & 0.97 \\
\hline CCL-5 & $1.60 \pm 1.09$ & $1.36 \pm 1.35$ & 0.75 & $1.36 \pm 0.91$ & $1.30 \pm 0.88$ & 0.90 \\
\hline sCD40L & $9.19 \pm 3.89$ & $9.43 \pm 3.74$ & 0.91 & $7.58 \pm 4.33$ & $4.63 \pm 1.62$ & $0.03^{\mathrm{b}}$ \\
\hline TNF- $\alpha$ & $2.85 \pm 3.86$ & $6.12 \pm 6.25$ & 0.19 & $5.82 \pm 10.78$ & $2.94 \pm 3.87$ & 0.32 \\
\hline TRAIL & $40.17 \pm 21.29$ & $38.21 \pm 12.62$ & 0.83 & $35.85 \pm 16.80$ & $101.24 \pm 145.69$ & 0.44 \\
\hline
\end{tabular}

$\mathrm{D}$, Venous invasion

\begin{tabular}{|c|c|c|c|c|c|c|}
\hline \multirow[b]{2}{*}{ Cytokines $^{\mathrm{a}}$} & \multicolumn{3}{|c|}{ Pre-CRT } & \multicolumn{3}{|c|}{ Post-CRT } \\
\hline & Absent & Present & P-value & Absent & Present & P-value \\
\hline IFN- $\gamma$ & $0.52 \pm 0.83$ & $0.36 \pm 0.64$ & 0.53 & $0.28 \pm 0.45$ & $0.28 \pm 0.41$ & 1.00 \\
\hline IL-10 & $1.10 \pm 1.61$ & $1.08 \pm 1.40$ & 0.98 & $0.87 \pm 1.40$ & $1.21 \pm 1.35$ & 0.48 \\
\hline IL-2 & $2.26 \pm 4.64$ & $2.52 \pm 4.08$ & 0.91 & $1.84 \pm 3.94$ & $2.01 \pm 3.16$ & 0.90 \\
\hline IL-4 & $7.76 \pm 12.71$ & $7.71 \pm 9.56$ & 0.99 & $5.46 \pm 7.04$ & $7.78 \pm 9.98$ & 0.43 \\
\hline IL-6 & $8.97 \pm 11.44$ & $10.67 \pm 8.61$ & 0.64 & $36.53 \pm 94.33$ & $31.51 \pm 44.51$ & 0.87 \\
\hline MCP-1 & $30.51 \pm 23.70$ & $39.61 \pm 20.85$ & 0.25 & $33.02 \pm 39.86$ & $36.93 \pm 23.16$ & 0.74 \\
\hline CCL-5 & $1.40 \pm 1.08$ & $1.68 \pm 1.13$ & 0.46 & $0.97 \pm 0.50$ & $1.60 \pm 1.02$ & $0.02^{\mathrm{b}}$ \\
\hline sCD40L & $10.91 \pm 3.83$ & $8.09 \pm 3.45$ & $0.04^{\mathrm{b}}$ & $7.10 \pm 3.11$ & $7.34 \pm 4.88$ & 0.86 \\
\hline TNF- $\alpha$ & $3.16 \pm 4.40$ & $3.27 \pm 4.18$ & 0.94 & $3.03 \pm 4.12$ & $7.12 \pm 12.65$ & 0.18 \\
\hline TRAIL & $41.46 \pm 21.87$ & $39.11 \pm 20.16$ & 0.77 & $56.38 \pm 80.91$ & $35.57 \pm 15.56$ & 0.38 \\
\hline
\end{tabular}

${ }^{\mathrm{a}}$ Data are expressed as mean $\mathrm{pg} / \mathrm{ml} \pm \mathrm{SD}$, except for CCL-5 and sCD40L, expressed as mean $\mathrm{ng} / \mathrm{ml} \pm \mathrm{SD}$; ${ }^{\mathrm{b}}$ statistical significance. Pre-CRT, sample collected prior to chemoradiation therapy (CRT); post-CRT, sample collected following CRT; IFN, interferon; IL, interleukin; MCP, monocyte-chemoattractant protein; CCL, chemokine ligand; sCD40L, soluble CD40-ligand; TNF, tumor necrosis factor; TRAIL, tumor necrosis factor-related apoptosis inducing ligand. 


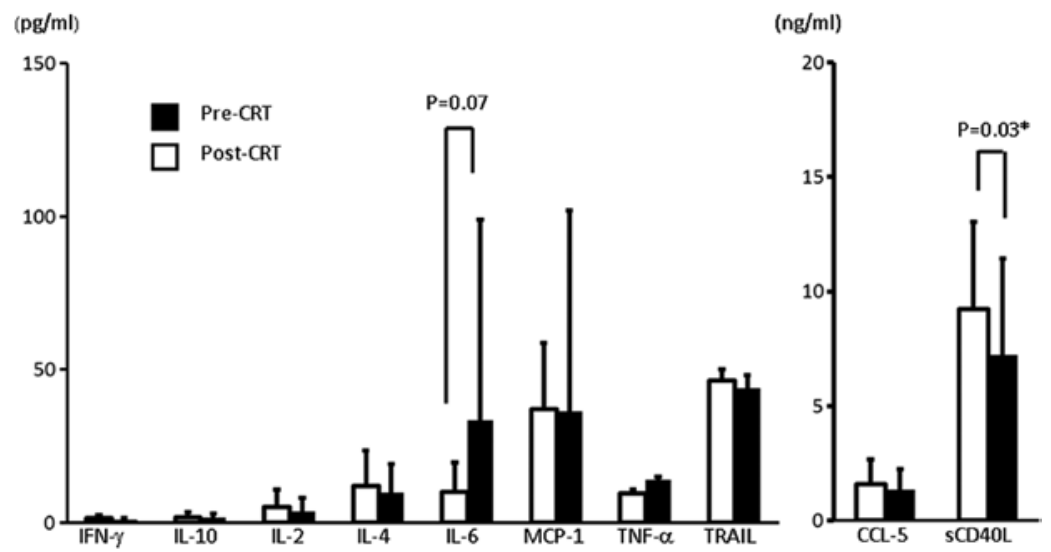

Figure 1. Concentration of the serum cytokines in pre-chemoradiation therapy (CRT) (filled bars) and in post-CRT samples (open bars). Data are expressed as mean \pm SD of results. "Statistical significance.

Table IV. Correlations between the pathological response and the cytokine levels.

\begin{tabular}{|c|c|c|c|c|c|c|}
\hline \multirow[b]{2}{*}{ Cytokines $^{\mathrm{a}}$} & \multicolumn{3}{|c|}{ Pre-CRT } & \multicolumn{3}{|c|}{ Post-CRT } \\
\hline & Low-R & High-R & $\mathrm{P}$-value & Low-R & High-R & P-value \\
\hline IFN- $\gamma$ & $0.51 \pm 0.82$ & $0.33 \pm 0.59$ & 0.46 & $0.37 \pm 0.48$ & $0.18 \pm 0.33$ & 0.19 \\
\hline IL-10 & $1.31 \pm 1.76$ & $0.85 \pm 1.08$ & 0.36 & $1.18 \pm 1.41$ & $0.97 \pm 1.33$ & 0.65 \\
\hline IL-2 & $3.36 \pm 5.27$ & $1.51 \pm 2.64$ & 0.20 & $2.48 \pm 3.94$ & $1.37 \pm 2.83$ & 0.34 \\
\hline IL-4 & $8.39 \pm 12.24$ & $7.03 \pm 9.23$ & 0.71 & $8.20 \pm 11.13$ & $5.4 \pm 5.63$ & 0.36 \\
\hline IL-6 & $11.14 \pm 11.59$ & $8.49 \pm 7.28$ & 0.38 & $50.12 \pm 82.94$ & $11.42 \pm 17.57$ & $0.03^{\mathrm{b}}$ \\
\hline MCP-1 & $38.38 \pm 24.50$ & $33.42 \pm 19.81$ & 0.51 & $37.63 \pm 25.88$ & $32.97 \pm 35.30$ & 0.67 \\
\hline CCL-5 & $1.53 \pm 1.18$ & $1.62 \pm 1.05$ & 0.76 & $1.43 \pm 0.95$ & $1.26 \pm 0.86$ & 0.59 \\
\hline sCD40L & $8.68 \pm 3.35$ & $9.78 \pm 4.29$ & 0.47 & $7.65 \pm 4.49$ & $6.80 \pm 3.98$ & 0.56 \\
\hline TNF- $\alpha$ & $4.31 \pm 4.99$ & $2.08 \pm 2.91$ & 0.11 & $9.11 \pm 13.28$ & $1.65 \pm 2.19$ & $0.03^{\mathrm{b}}$ \\
\hline TRAIL & $40.50 \pm 22.34$ & $39.47 \pm 19.21$ & 0.89 & $56.85 \pm 71.76$ & $31.20 \pm 15.79$ & 0.18 \\
\hline
\end{tabular}

${ }^{a}$ Data are expressed as mean $\mathrm{pg} / \mathrm{ml} \pm \mathrm{SD}$, except for CCL-5 and sCD40L, expressed as mean $\mathrm{ng} / \mathrm{ml} \pm \mathrm{SD}$; bindicates statistical significance. Pre-CRT, sample collected prior to chemoradiation therapy (CRT); post-CRT, sample collected following CRT; Low-R, low response; High-R, high response; IFN, interferon; IL, interleukin; MCP, monocyte-chemoattractant protein; CCL, chemokine ligand; sCD40L, soluble CD40-ligand; TNF, tumor necrosis factor; TRAIL, tumor necrosis factor-related apoptosis inducing ligand.
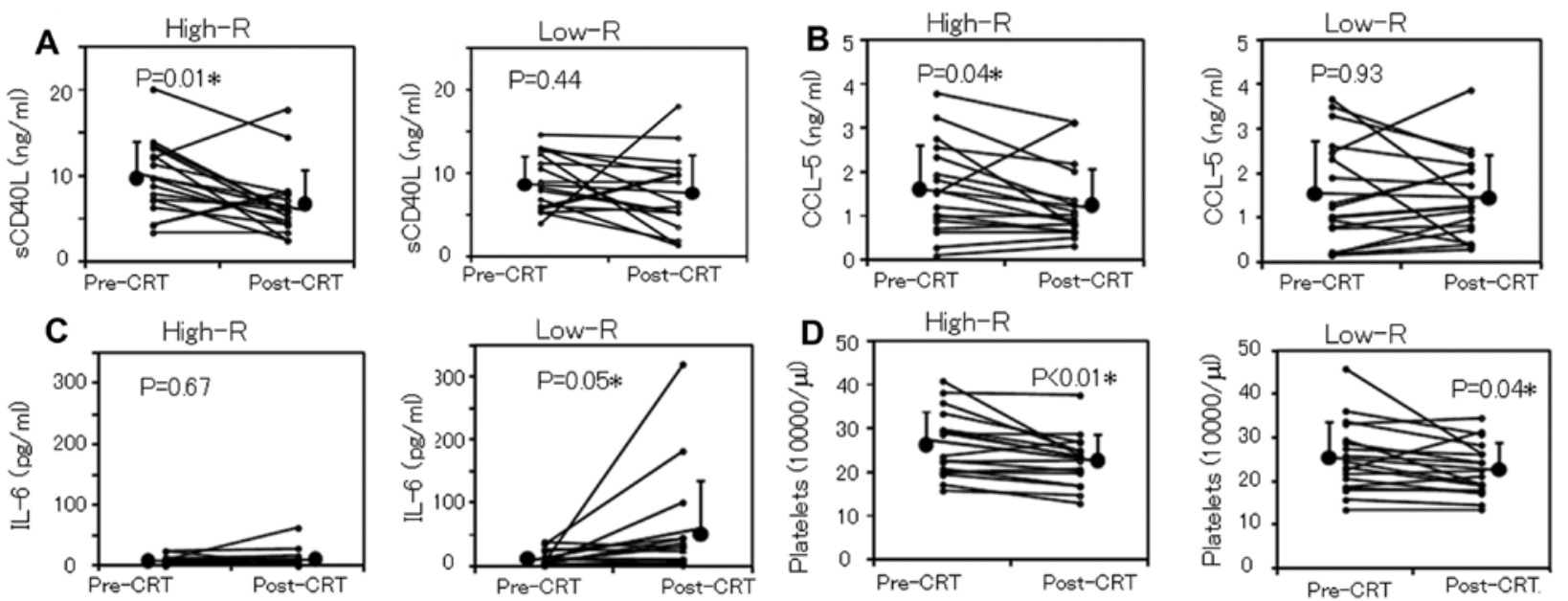

Figure 2. The pre- to post-chemoradiation therapy (CRT) changes in the concentration of the various cytokine/chemokines in high response (High-R) and in low response (Low-R). Each line in the plots represents the values obtained from the same patient pre- and post-CRT. The mean \pm SD value is also shown. (A) The soluble CD40-ligand (sCD40L) and (B) CCL-5 (RANTES) levels were significantly reduced following CRT in the High-R, but not in the Low-R, whereas (C) interleukin (IL)-6 levels were significantly increased following CRT in the Low-R, but not in the High-R. (D) Platelet counts were significantly reduced in both High- $\mathrm{R}$ and Low- $\mathrm{R}$, but the difference was more evident in the High- $\mathrm{R}(\mathrm{P}<0.01$ and $\mathrm{P}=0.04$, respectively). 

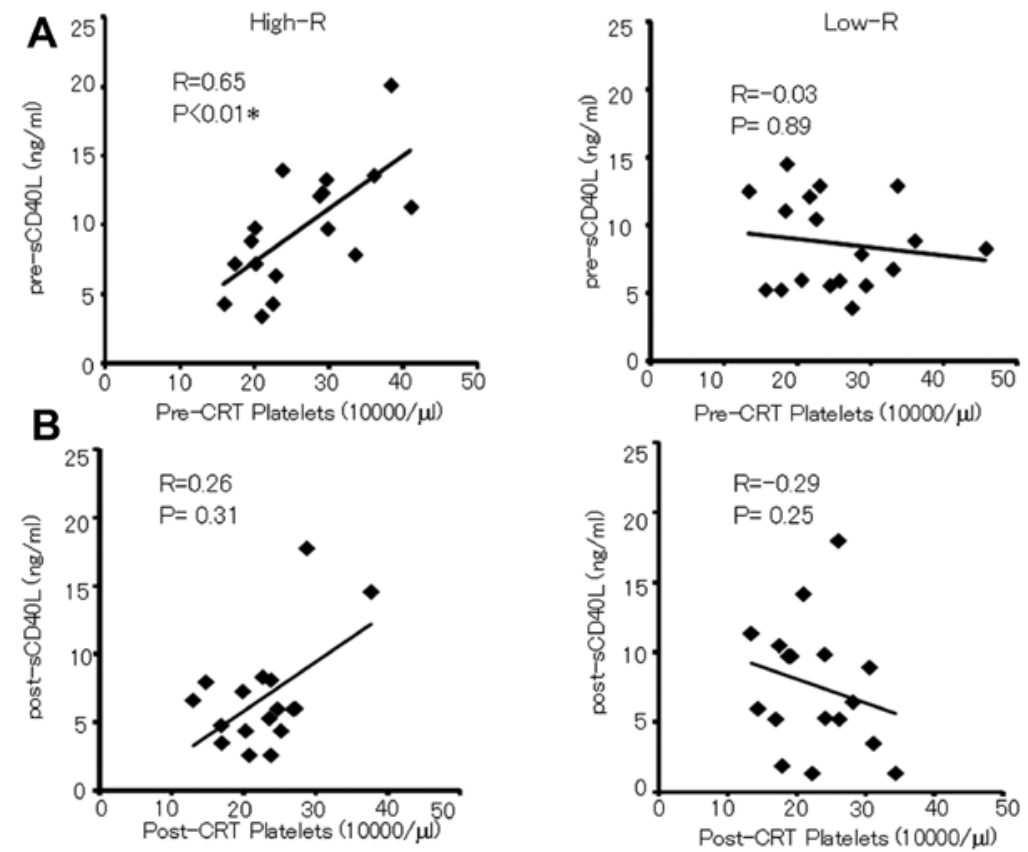

Figure 3. Correlation between pre-/post-chemoradiation therapy (CRT) soluble CD40-ligand (sCD40L) and platelet counts in high response (High-R) and in low response (Low-R). (A) The pre-CRT sCD40L level significantly correlated with pre-CRT platelet counts in the High-R, but not in the Low-R. (B) The post-CRT sCD40L level had no significant correlation with the post-CRT platelet counts in either the High-R, or the Low-R.

Table V. Correlations between the pathological response and the leukocyte subsets.

\begin{tabular}{|c|c|c|c|c|c|c|}
\hline \multirow[b]{2}{*}{ Leukocyte subset ${ }^{\mathrm{a}}$} & \multicolumn{3}{|c|}{ Pre-CRT } & \multicolumn{3}{|c|}{ Post-CRT } \\
\hline & Low-R & High-R & P-value & Low-R & High-R & P-value \\
\hline T-lymphocytes & $973 \pm 217$ & $1197 \pm 419$ & 0.05 & $555 \pm 230$ & $609 \pm 405$ & 0.63 \\
\hline B-lymphocytes & $134 \pm 99$ & $201 \pm 163$ & 0.16 & $31 \pm 19$ & $42 \pm 40$ & 0.31 \\
\hline NK cells & $260 \pm 243$ & $317 \pm 229$ & 0.49 & $199 \pm 135$ & $191 \pm 151$ & 0.86 \\
\hline MDSCs & $1.32 \pm 1.35$ & $2.11 \pm 2.64$ & 0.31 & $3.51 \pm 5.72$ & $3.18 \pm 7.43$ & 0.91 \\
\hline
\end{tabular}

${ }^{\mathrm{a} D a t a}$ are expressed as mean $($ cells $/ \mu \mathrm{l}) \pm \mathrm{SD}$, except for MDSCs, expressed as mean $\% \pm \mathrm{SD}$. Pre-CRT, sample collected prior to chemoradiation therapy (CRT); post-CRT, sample collected following CRT; Low-R, low response; High-R, high response; NK cells, natural killer cells; MDSCs, myeloid-derived suppressor cells .

kines and inflammatory cells. Both the innate and the adaptive immune responses are orchestrated by cytokines, which establish the most efficient machinery for preventing cancer growth and progression. By contrast, a number of cytokines might have inhibitory effects on this machinery, leading to the promotion of tumor growth (6,9-11).

To date, numerous studies have been conducted to evaluate the prognostic factors of $\mathrm{RC}$ patients receiving CRT, but only few reports have focused on the plasma cytokines, such as IL-6, TGF- $\beta$, IL-10 and sCD40L, and the reported findings on their correlation with the response to CRT have been ambiguous (4,13-15). In prostate cancer patients receiving radiotherapy, increased levels of IL-6, but not IL-2, IL-4, or TNF- $\alpha, 15$ days following radiotherapy (RT), were found to correlate with a marked decrease in PSA level (16). Other cytokines, such as IL- 1 and TGF- $\beta$, have also been reported to be affected by RT in prostate cancer patients. Therefore, it is possible that levels of some cytokines in patient plasma might correlate with the effects of CRT and, thus, may be useful for the prediction of the response to therapy.

First, we analyzed the correlation between the plasma levels of various cytokines and the clinicopathological features of RC patients treated with CRT and surgery. We observed a significant correlation between the pre-CRT plasma levels of sCD40L and the depth of tumor invasion and venous invasion, with SCD40L levels being significantly increased in cases of downsizing of the primary tumor (ypT0-2) compared to locally advanced tumors (ypT3-4) following CRT. Moreover, the pre-CRT sCD40L level was significantly higher in cases without venous invasion compared to those with venous invasion. In addition, the post-CRT level of sCD40L was significantly higher in the group without lymphatic invasion, compared to the group with lymphatic invasion. By contrast, the post-CRT level of CCL-5 was significantly higher in locally advanced tumors (ypT3-4) compared to cases of downsizing of the primary tumor (ypT02 ), and also significantly higher in those cases with venous 
invasion, compared to the cases without. Thus, it appears that an increased level of CCL-5 following CRT is associated with a poor response to therapy, while a higher pre-CRT level of sCD40L is associated with downsizing of the primary tumor.

Next, we analyzed the correlation of the plasma levels of cytokines and the pathological response to therapy, and found a significant decrease in the plasma levels of SCD40L in postCRT compared to pre-CRT samples, but when High-R and Low-R groups were analyzed separately, a significant reduction in SCD40L following CRT was observed only in High-R. By contrast, although without statistical significance, the postCRT level of IL- 6 was higher than the pre-CRT one. When changes in IL- 6 were analyzed in the High-R and Low-R groups separately, the significant increase was observed in the Low-R, but not in the High-R. By contrast, a significant decrease in the level of CCL-5 following CRT was observed in the High-R, but not in the Low-R. The other cytokines analyzed, namely IL-2, -4, -10 , INF- $\gamma$, TNF- $\alpha$, TRAIL, MCP-1 and CCL- 5 were not affected by CRT.

Since platelets and activated T-lymphocytes are known to express surface CD40L and to generate a soluble fragment of CD40L (17,18), we investigated whether platelet counts were affected by CRT, and observed that platelets were significantly reduced in both High-R and Low-R, but the difference was more evident in the High-R. Similarly, a significant reduction in the levels of sCD40L and CCL-5 following CRT was observed, but only in the High-R. Thus, it seems that the significant reduction in SCD40L and CCL-5 following CRT is associated with a favorable response to therapy. It is possible that changes in these cytokines merely reflect changes in the platelet counts. However, the significant correlation between pre-CRT sCD40L and platelet counts was observed only in High-R, and in this group, a negative correlation between pre-CRT CCL-5 and platelet counts was found, potentially leading to a negative correlation between pre-CRT sCD40L and CCL-5. Thus, it may be speculated that, although both cytokines are significantly reduced following CRT in High-R, they have antagonistic effects in the response to CRT. Further corroborating this hypothesis is our finding that the higher pre-CRT level of sCD40L was associated with tumor downstaging following CRT and with absence of venous invasion, whereas higher post-CRT CCL-5 was associated with locally more advanced tumors and the presence of venous invasion.

In our previous study (Tada et al, unpublished data), we demonstrated that, compared to pre-CRT, the postCRT numbers of T- and B-lymphocytes were significantly decreased, and the patients with a favorable response (High-R) had a higher number of pre-CRT T-lymphocytes than those with a poor response (Low-R group) $(\mathrm{P}<0.01)$. In the present study however, we were unable to demonstrate a clear association between the investigated cytokines and the immune cells. Recently, in addition to the antitumor immunity-inducing effect, the immunosuppressive effect of the CD40-CD40L costimulator pathway, possibly via stimulation of MDSCs, was reported $(19,20)$. More recently, tumor-infiltrating monocytic MDSCs have been shown to secrete CCL-5, recruiting Tregs, which favor tumor growth in mice (21). In non-small cell lung cancer patients treated with EGFR-TKIs, low levels of CCL-5 at diagnosis were reported to be significantly associated with long-term survival (22). In our series, however, we did not find any correlation between sCD40L in plasma and the populations of MDSCs [CD33(+)HLA(-)DR(-)], either pre- or post-CRT, in either High-R or Low-R. However, a tendency for a negative correlation between post-CRT levels of CCL-5 and CD3(+) T-cell counts was observed in the High-R, whereas a tendency for a positive correlation was observed in the Low-R. Thus, it is tempting to speculate that Tregs, characterized as CD4(+)/CD25(+)/FoxP3(+), are involved in the poor response of Low-R, possibly by recruiting Tregs. CD4(+) cells are part of CD3(+) cells, which showed a tendency for a positive correlation with CCL-5, and this cytokine is reported to be associated with progression and metastasis (23-26) and with poor response to therapy in various tumor types $(27,28)$. In addition, the negative correlation between CCL-5 and CD3(+) T-cells in the High-R strengthens our hypothesis. Unfortunately, in this study, we did not investigate Tregs, and this is the topic of a future study.

The reason why post-CRT sCD40L levels are significantly reduced in High-R is not known, but it is known that, in addition to the immunomodulatory effects, the CD40 signaling pathway also plays an important role in proliferation, differentiation and apoptosis of cancer cells. Many tumor cells also express CD40 (29-32), and evidence suggests that ligation of CD40 might promote either cell proliferation or apoptosis, depending on the intensity of CD40L signaling $(33,34)$. Although we did not investigate the expression of CD40 in cancer cells, it can be speculated that SCD40L may induce antitumor effects, either through binding to immune cells, stimulating an antitumor immunity, or through the direct binding to cancer cells, inducing their apoptotic cell death; in both situations, sCD40L levels are reduced by consumption.

In addition, in our series, we found higher levels of postCRT IL- 6 and TNF- $\alpha$ in the Low-R compared to the High-R, which suggests that these cytokines might serve as indicators of a poor response to CRT. Also, when comparing pre- and post-CRT levels of these cytokines, a significant increase in post-CRT IL-6 was observed in the Low-R, corroborating this hypothesis. IL-6 levels were also reported to be associated with poor prognosis in various types of cancer, including pancreatic, breast, ovarian and esophageal $(15,35)$, and increased levels of TNF- $\alpha$ have been shown to correlate with poor prognosis in colorectal cancer patients $(36,37)$. IL-6 has been shown to be associated with tumor stage, metastasis and survival of colorectal cancer patients (13-15,35). Furthermore, increased levels of serum IL- 6 during CRT have been reported, but no association with the response rate has been demonstrated (4). In our series, 3 cases in the Low-R showed a significant elevation in IL- 6 following CRT, which resulted in a significant increase in the post-CRT levels compared to the pre-CRT ones in this group of patients. On the other hand, the IL-6 levels did not change following CRT in the High-R. From these data, we hypothesize that IL-6, as well as TNF- $\alpha$, reflect the inflammatory damage caused by CRT, which might impair antitumor immunity. Thus, IL- 6 and TNF- $\alpha$ might be useful markers of a poor response to CRT, but since only the post-CRT, but not the pre-CRT levels, correlated with the response to treatment, their use as predictive markers prior to therapy is uncertain.

In a recent report, we demonstrated that the pre-CRT platelet counts correlated with the response of CRT and the 
local recurrence-free survival in RC patients (38). When patients were divided into thrombocytotic (defined as platelet count $>36.5 \times 10,000 / \mu l$ ) and those with platelet counts within the normal range, those with thrombocytosis had a poor response to CRT (38). In the present series, only two patients were thrombocytotic according to this classification, and one case classified as High-R and the other as Low-R. Interestingly, we observed that the platelet counts were significantly affected by CRT in both High-R and Low-R, but the difference was more evident in the High-R. This result might merely reflect the association of thrombocytosis with a poor response to CRT, but considering that platelets are the major source of both $\mathrm{SCD} 40 \mathrm{~L}$ and CCL-5, it is highly probable that platelets play an important role in the complex interaction between cytokines and the immune system, by eliciting antitumor immunity. Considering all these data, it is tempting to speculate that patients in a better immunological condition prior to treatment, including higher blood platelet counts, are able to induce more effective antitumor immunity, and SCD40L might contribute to the antitumor effects, whereas CCL-5 might inhibit it. Whether platelets and immune cells preferentially produce either SCD40L or CCL-5 might determine the fate of cancer cells.

In conclusion, our results suggest that the complex interaction among the immune system, platelets and tumor cells, mediated through various cytokines/chemokines, determines the response of RC patients to CRT. The analysis of the immunological condition of the patient prior to treatment, including immune cells and platelets, might help predicting the response to treatment, and the pre-/post-CRT measurement of sCD40L, CCL-5, TNF- $\alpha$ and IL-6 may also be useful, particularly in deciding on the need of additional therapy. Since the number of cases included in this study was relatively small, a more large-scale prospective study will be necessary to confirm the present findings, and to clarify this complex association between the immune system, platelets and cancer cells, cross-linked through various cytokines/chemokines. By understanding the role of these molecules in regulating the response to CRT, new approaches for the improvement of treatment might be proposed.

\section{Acknowledgements}

The authors thank Ms. Mika Matsuhashi, Mr. Yutaka Nagura and Ms. Junko Iino from the Department of Transfusion Medicine (University of Tokyo) for their kind advice and technical assistance.

\section{References}

1. Sauer R, Becker H, Hohenberger W, et al: Preoperative versus postoperative chemoradiotherapy for rectal cancer. N Eng J Med 351: 1731-1740, 2004.

2. Bosset JF, Collette L, Calais G, et al: Chemotherapy with preoperative radiotherapy in rectal cancer. N Eng J Med 355: 1114-1123, 2006

3. Ortholan C, Francois E, Thomas O, et al: Role of radiotherapy with surgery for T3 and resectable T4 rectal cancer: evidence from randomized trials. Dis Colon Rectum 49: 302-310, 2006.

4. Debucquoy A, Goethals L, Geboes K, Roels S, Mc Bride WH and Haustermans K: Molecular responses of rectal cancer to preoperative chemoradiation. Radiother Oncol 80: 172-177, 2006.
5. Baier PK, Eggstein S, Wolff-Vorbeck G, Baumgartner U and Hopt UT: Chemokines in human colorectal carcinoma. Anticancer Res 25: 3581-3584, 2005.

6. Dranoff G: Cytokines in cancer pathogenesis and cancer therapy (Review). Nat Rev Cancer 4: 11-22, 2004.

7. Okita R, Yamaguchi Y,Ohara M, et al: Targeting of $\mathrm{CD} 4{ }^{+} \mathrm{CD} 25^{\text {high }}$ cells while preserving $\mathrm{CD} 4{ }^{+} \mathrm{CD} 25^{\text {low }}$ cells with low-dose chimeric anti-CD25 antibody in adoptive immunotherapy of cancer. Int J Oncol 34: 563-572, 2009.

8. Ostrand-Rosenberg S: Myeloid-derived suppressor cells: more mechanisms for inhibiting antitumor immunity (Review). Cancer Immunol Immunother 59: 1593-1600, 2010.

9. McDonnell AM, Nowak AK and Lake RA: Contribution of the immune system to the chemotherapeutic response. Semin Immunopathol 33: 353-367, 2011.

10. Ong ZY, Gibson RJ, Bowen JM, et al: Pro-inflammatory cytokines play a key role in the development of radiotherapyinduced gastrointestinal mucositis. Radiat Oncol 5: 22, 2010.

11. Schaue D, Kachikwu EL and McBride WH: Cytokines in radiobiological responses: a review (Review). Radiat Res 178: 505-523, 2012.

12. Lin WW and Karin M: A cytokine-mediated link between innate immunity, inflammation, and cancer. J Clin Invest 117: 1175-1183, 2007.

13. Lin CC, Liu CY, Chen MJ, et al: Profiles of circulating endothelial cells and serum cytokines during adjuvant chemoradiation in rectal cancer patients. Clin Transl Oncol 15: 855-860, 2013.

14. Chung YC and Chang YF: Serum interleukin-6 levels reflect the disease status of colorectal cancer. J Surg Oncol 83: 222-226, 2003.

15. Waldner MJ, Foersch S and Neurath MF: Interleukin-6 - a key regulator of colorectal cancer development. Int J Biol Sci 8: 1248-1253, 2012.

16. Lopes $\mathrm{CO}$ and Callera F: Three-dimensional conformal radiotherapy in prostate cancer patients: rise in interleukin 6 (IL-6) but not IL-2, IL-4, IL-5, tumor necrosis factor- $\alpha$, MIP-1- $\alpha$, and LIF levels. Int J Radiat Oncol Biol Phys 82: 1385-1388, 2012.

17. Andre P, Nannizzi-Alaimo L, Prasad SK and Phillips DR: Platelet-derived CD40L: the switch-hitting player of cardiovascular disease. Circulation 106: 896-899, 2002.

18. Aukrust P, Damas JK and Solum NO: Soluble CD40 ligand and platelets: self-perpetuating pathogenic loop in thrombosis and inflammation? J Am Coll Cardiol 43: 2326-2328, 2004.

19. Schlom J, Jochems C, Gulley JL and Huang J: The role of soluble CD40L in immunosuppression. Oncoimmunology 2: e22546, 2013.

20. Huang J, Jochems C, Talaie T, et al: Elevated serum soluble CD40 ligand in cancer patients may play an immunosuppressive role. Blood 120: 3030-3038, 2012.

21. Schlecker E, Stojanovic A, Eisen C, et al: Tumor-infiltrating monocytic myeloid-derived suppressor cells mediate CCR5dependent recruitment of regulatory $\mathrm{T}$ cells favoring tumor growth. J Immunol 189: 5602-5611, 2012.

22. Umekawa K, Kimura T, Kudoh S, et al: Plasma RANTES, IL-10, and IL-8 levels in non-small-cell lung cancer patients treated with EGFR-TKIs. BMC Res Notes 6: 139, 2013.

23. Lin S, Wan S, Sun L, et al: Chemokine C-C motif receptor 5 and $\mathrm{C}-\mathrm{C}$ motif ligand 5 promote cancer cell migration under hypoxia. Cancer Sci 103: 904-912, 2012.

24. Chuang JY, Yang WH, Chen HT, et al: CCL5/CCR5 axis promotes the motility of human oral cancer cells. J Cell Physiol 220: 418-426, 2009

25. Lapteva $\mathrm{N}$ and Huang XF: CCL5 as an adjuvant for cancer immunotherapy. Expert Opin Biol Ther 10: 725-733, 2010.

26. Zhang Y, Yao F, Yao X, et al: Role of CCL5 in invasion, proliferation and proportion of $\mathrm{CD}^{4} 4^{+} / \mathrm{CD} 24^{-}$phenotype of $\mathrm{MCF}-7$ cells and correlation of CCL5 and CCR5 expression with breast cancer progression. Oncol Rep 21: 1113-1121, 2009.

27. Yi EH, Lee CS, Lee JK, et al: STAT3-RANTES autocrine signaling is essential for tamoxifen resistance in human breast cancer cells. Mol Cancer Res 11: 31-42, 2013.

28. Radziwon-Balicka A, Medina C, O'Driscoll L, et al: Platelets increase survival of adenocarcinoma cells challenged with anticancer drugs: mechanisms and implications for chemoresistance. Br J Pharmacol 167: 787-804, 2012.

29. Li R, Chen WC, Pang XQ, Hua C, Li L and Zhang XG: Expression of CD40 and CD40L in gastric cancer tissue and its clinical significance. Int J Mol Sci 10: 3900-3917, 2009.

30. Roselli M, Mineo TC, Basili S, et al: Soluble CD40 ligand plasma levels in lung cancer. Clin Cancer Res 10: 610-614, 2004. 
31. Pan W, Gong J, Yang C, et al: Peripheral blood CD40-CD40L expression in human breast cancer. Ir J Med Sci, Feb 28, 2013 (Epub ahead of print).

32. Toutirais $\mathrm{O}$, Gervais A, Cabillic F, et al: Effects of CD40 binding on ovarian carcinoma cell growth and cytokine production in vitro. Clin Exp Immunol 149: 372-377, 2007.

33. Li R, Chen WC, Pang XQ, Tian WY and Zhang XG: Influence of sCD40L on gastric cancer cell lines. Mol Biol Rep 38: 5459-5464, 2011.

34. Mathur RK, Awasthi A, Wadhone P, Ramanamurthy B and Saha B: Reciprocal CD40 signals through p38MAPK and ERK-1/2 induce counteracting immune responses. Nat Med 10: 540-544, 2004

35. Becker C, Fantini MC, Wirtz S, et al: IL-6 signaling promotes tumor growth in colorectal cancer. Cell Cycle 4: 217-220, 2005.
36. Ardestani S, Li B, Deskins DL, Wu H, Massion PP and Young PP: Membrane versus soluble isoforms of TNF- $\alpha$ exert opposing effects on tumor growth and survival of tumor-associated myeloid cells. Cancer Res 73: 3938-3950, 2013.

37. Ferroni P, Riondino S, Portarena I, et al: Association between increased tumor necrosis factor alpha levels and acquired activated protein $C$ resistance in patients with metastatic colorectal cancer. Int J Colorectal Dis 27: 1561-1567, 2012.

38. Kawai K, Kitayama J, Tsuno NH, Sunami E and Watanabe T: Thrombocytosis before pre-operative chemoradiotherapy predicts poor response and shorter local recurrence-free survival in rectal cancer. Int J Colorectal Dis 28: 527-535, 2013. 